Case Report

iMedPub Journals

www.imedpub.com

Vol.2 No.4:29

DOI: $10.21767 / 2573-4482.100062$

\section{A Case of Orchiectomy after Femoral Artery PTA}

\section{Abstract}

High femoral arterial puncture above the groin ligament may cause different types of complications as pseudo aneurysm, hemorrhage involving retroperitoneal region, and groin region hematoma. We present a case of 70 years old man who was admitted to our Hospital and underwent left superficial femoral PTA. The post- procedural course was complicated by hematoma in the groin site of puncture, which led to the formation of a giant hematoma rapidly infiltrating the scrotal and right groin zone. It required surgical revision of the puncture site, evacuation of the hematoma, and radical right orchiectomy. The case reported suggests that patients in whom a hematoma in scrotal region arises after PTA should immediately accompanied into the operating room for a surgical evacuation without undergoing only to medical treatment because an early surgical treatment could reveal fundamental for organ spearing.

Keywords: PTA; Surgery; Complication; Orchiectomy

Received: September 26, 2017; Accepted: October 16, 2017; Published: October 23, 2017

\section{Introduction}

High femoral arterial puncture above the groin ligament can cause pseudo aneurysm, hemorrhage involving retroperitoneal region, groin region, spermatic cord, and scrotum [1,2]. However, the testicular infarction caused by extrinsic compression is a very rare complication of percutaneous procedures. In literature, only four cases of testicular ischemic necrosis are described [3-6], but a giant hematoma with serious stuffing of the spermatic cord and the testis has been never reported.

\section{Case Presentation}

A 70 years old man was admitted to our Hospital with diagnosis of peripheral obstructive arterial disease of the lower limbs. He was symptomatic for claudicatio intermittens with 30-meater freedom walking in plane. In his past medical history were previous myocardial infarction treated by quadruple coronary artery bypass, moderate aortic valve stenosis, moderate mitral insufficiency, cardiac ejection fraction $25 \%$, atrial fibrillation, carotid artery disease with intimal sclerosis without stenosis, type II diabetes in medical therapy, stage II chronic kidney disease, and HCV - related liver disease. Patient underwent angiography of the lower limbs, which showed long, calcific, and eccentric stenosis of the left superficial femoral artery treated by percutaneous trans luminal angioplasty (PTA) as showed in Figure 1. The post- procedural course was complicated by giant

\section{Francesca D'Auria ${ }^{1,2 *}$, Giulio Tessitore ${ }^{1}$, Nicola Avossa ${ }^{1}$ and Mario Fabbrocini ${ }^{1}$}

1 Cardiovascular Department, Istituto Clinico Città di Alessandria - Policlinico di Monza, Alessandria, Via Moccagatta 30, 15121 Alessandria, Italy

2 Cardiovascular Surgery Department, Campus Bio - Medico University of Rome, Via Alvaro del Portillo 200, 00128 Rome, Italy

*Corresponding author: Francesca D'Auria

”f.dauria@hotmail.com

Cardiovascular Surgery Department, Campus Bio- Medico University of Rome, Via Alvaro del Portillo n. 200, 00128 Rome, Italy.

Tel: 00393337246468

Citation: D'Auria F, Tessitore G, Avossa N, Fabbrocini M (2017) A case of orchiectomy after femoral artery PTA. J Vasc Endovasc Surg. Vol.2 No.4:29
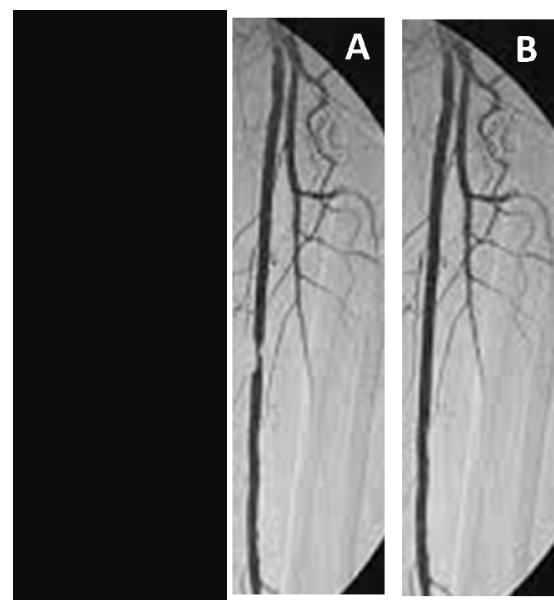

Figure 1 Left superficial femoral artery angiography (A) and PTA result (B). 
hematoma in the groin in correspondence of the puncture site. A Doppler examination revealed a left common femoral artery pseudo aneurysm, so a pressure dressing was positioned. After 5 hours from the procedure, the hematoma was expanded infiltrating the left groin and the left scrotum. The hematoma required surgical revision of the puncture site and its evacuation. Left orchiectomy was also necessary because the hematoma had seriously filled the spermatic cord and the testis (Figure 2), irreversibly damaging the organ (Figure 3).

\section{Discussion}

Testicular ischemia is a rarely complication with only four cases till now reported in the literature [3-6]. After lowerlimb peripheral PTA, the ischemic complications range from intermittent claudication to ischemic necrosis requiring rePTA, or surgical bypass, or amputation. Testicular ischemia is a rarely reported complication with only three cases hereto reported in the literature. The mechanism of testicular ischemia

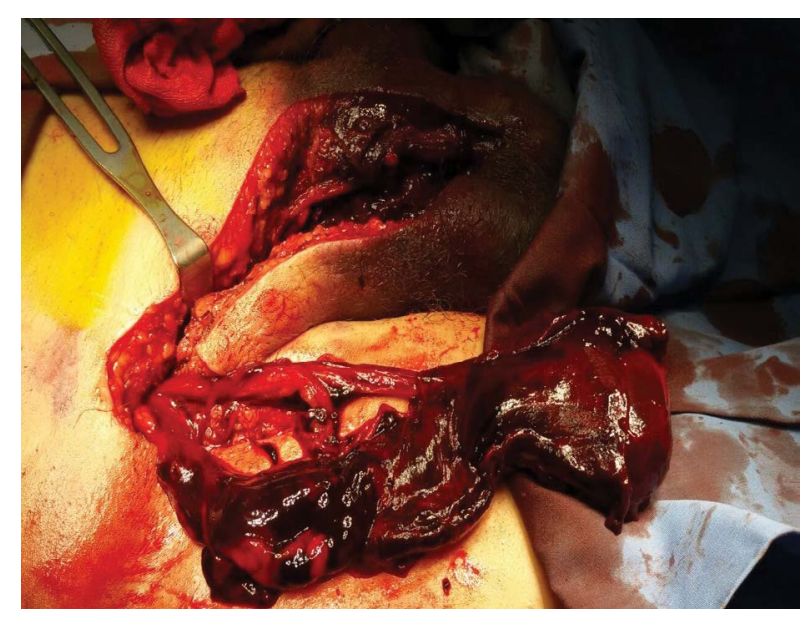

Figure 2 Left spermatic cord and left testis compressed and filled by blood.

\section{References}

1 Jackson EA, Munir K, Schreiber T, Rubin JR, Cuff R, et al. (2014) Impact of sex on morbidity and mortality rates after lower extremity interventions for peripheral arterial disease: observations from the Blue Cross Blue Shield of Michigan Cardiovascular Consortium. J Am Coll Cardiol 63: 2525-2530.

2 Aziz A, Green A, Maskova J, Rajagopalan S, Bachoo P (2014) Pseudoaneurysm of the sural artery: a rare complication of lower limb angioplasty. Scott Med J 59: e7-e10.

3 McKenna AJ, Gambardella I, Collins A, Harkin DW (2009) Testicular

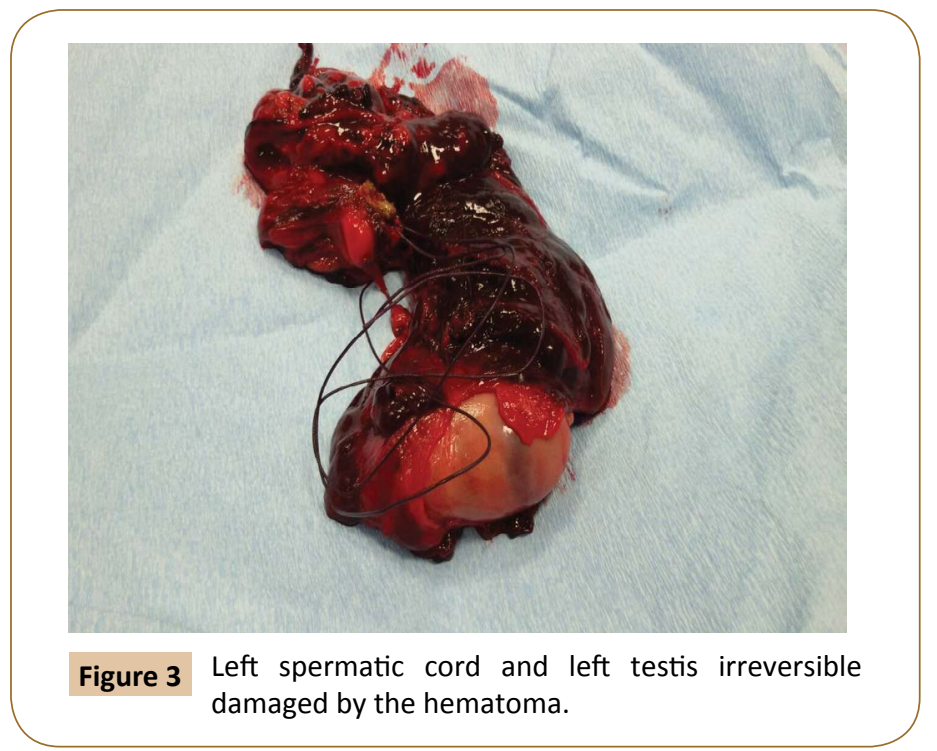

endovascular procedures is not well known. Possible recognized causes include graft migration, thromboembolic events, absence of collateral blood flow, and/or delayed occlusion of testicular arteries. This case reported suggests that external compression of the testicular arteries could immediately lead to catastrophic scenario, therefore patients in which a scrotal hematoma arises after PTA should immediately accompanied into the operating room for a surgical evacuation without undergoing only to medical treatment because an early surgical treatment could reveal fundamental for organ spearing. The hematoma evacuation is to be especially recommended in case of primitive blood coagulation problems, coagulopathy secondary to liver disease, atrial fibrillation for which it is immediately introduced anticoagulation after PTA, problems of coagulation and/or platelet aggregation system, i.e. in case of recent coronary DES implantation, and/or other clinical conditions indicating a greater propensity to bleed.

infarction: a rare complication of endovascular aneurysm repair treatment for aortoiliac aneurysm. J Vasc Surg 50: 1487-1489.

4 Finnerty N, Rancour S, King A (2014) Acute testicular ischemia following endovascular abdominal aortic aneurysm repair identified in the emergency department. Case Rep Emerg Med.

5 Pathmarajah T, Abdelhamid M, Tenna AS, Paton DJW, Hockley JA et al. (2017) Acute Global Testicular Infarction Post-EVAR from Cholesterol Embolisation can be mistaken for Torsion. EJVES Short Rep 35: 11-15.

6 Thomas E, Parra BL, Patel S (2017) Post-endovascular aneurysm repair (EVAR) testicular ischemia: A rare complication. Urol Case Rep 14: 35-37. 